\title{
IMPROVED DEMNTIA IMAGES DETECTION AND CLASSIFICATION USING TRANSFER LEARNING BASE CONVULATION MAPPING WITH ATTENTION LAYER AND XGBOOST CLASSIFIER
}

\author{
Ms. Harsimran Guram , Dr.Ashok Sharma \\ Lovely professional University \\ harsimran.679@gmail.com,drashoksharma@hotmail.co.in
}

Article History:Received:11 november 2020; Accepted: 27 December 2020; Published online: 05 April 2021

\begin{abstract}
A classification scheme for the etiology of brain disease based on magnetic resonance imaging is proposed in this paper. Then, attention-based, transfer learning (Extracting variable characteristics of patterns from MRI scans) was used to generate more accurate predictive patterns, and finally, features were trained and used to classify fMRI data to Boost derived hyper-parameters were obtained and evaluated to identify different patterns of dementia risk. Typically-to-to-independent variable extractions are performed by using gradient boosting and then produces derived variables. The MRI in the system is pulled from the ADNI database. When using Feature extractor's technique, we find that most features are extracted at an acceptable speeds. The experimental results proved that the proposed approach can be applied to the task of classifying output in the proper manner. The method would help to increase precision and accuracy by (almost) 4.2 percent, while keeping recall at (virtually) $94.6 \%$
\end{abstract}

Keywords: Dementia, classification, Attention, CNN, XG-Boost, transfer learning

\section{INTRODUCTION}

Nowadays, for those of the age 50 and up, Dementia has become a serious health concern. In Europe, about two and a half million new cases with dementia-related diagnoses are being made each year.

Dementia is a type of incremental and progressive dementia. It is not only seen in older people, but more of them have warned the researchers about the neuro-disorder that leads to a lack of memory and a later recall from memory. DIA may help patients slow it down to a certain degree if early diagnosis is possible. The majority of scientists claim that two proteins are predominant in the brain. Beta-Amyloid, an abnormal level, is betaamyloid which forms and becomes one of plaque that interfereses with cell activity Othe other is called tau, which often goes beyond the neuron's transport structure, and forms neurofibrillary tangles that disables neuron movement

According to an analysis of current and possible future demographics, there will be more than 120 million individuals with dementia by the year 2050, as well as 75 million.

If everybody was granted an equal chance, our disparities would almost completely vanish.

Today, Dementia has become one of the world's biggest health problems for people aged 50-70. About 2,5 million individuals were introduced to Europe's pool of dementia-related problems.

Indeed, dementia is a neurological condition that causes progressive and continuous memory loss. Researchers have observed that it effects a larger percentage of the elderly than young people, but has strengthened their interest to study neurodisorders resulting in memory loss[24]. Dementia can help the patient to calm down to some degree if diagnosed at an early stage. The majority of scientists claim that two proteins are predominant in the brain. Beta-Amyloid, an abnormal level, is beta-amyloid which forms and becomes one of plaque that interferes with cell activity other is called tau, which often goes beyond the neuron's transport structure, and forms neurofibrillary tangles that disables neuron movement

According to an analysis of current and possible future demographics, there will be more than 120 million individuals with dementia by the year 2050, as well as 75 million.

If everybody was granted an equal chance, our disparities would almost completely vanish. Dementia is a neurodegenerative disease which effects the normal functioning of patients and it starts effecting heart and lungs of patients leading to death of patient. Dementia is divided into following types of Neurodegenerative and nonneurodegenerative [18].Types of dementia is indicated in Table 1.1

Table 1.1: Types of Dementia [18]

\begin{tabular}{|l|l|}
\hline Neurodegenerative & Non-neurodegenerative \\
\hline Alzheimer's disease & Vascular dementia \\
\hline
\end{tabular}

*Corresponding author: Ms. Harsimran Guram

Lovely professional University

harsimran.679@gmail.com 


\begin{tabular}{|l|l|}
\hline Frontotemporal dementia & Normal pressure hydrocephalus \\
\hline Dementia with lewy bodies & Autoimmune causes \\
\hline Multiple system atrophy & Infectious causes \\
\hline Alcoholic cognitive impairment & Toxic causes \\
\hline Huntington disease & Vitamin deficiency \\
\hline
\end{tabular}

When this disease came into limelight, mostly specialist doctors examine the symptoms or cases of dementia cure by looking into below listed methodology:-

a) History of a person includes interview of a person with a neurologist

b) Mental state of person keeping focus on neurological test

c) Blood sample tests

d) Scan of a Brain was done to diagnose.

All these steps remain the baseline for diagnosing the patient to diagnose dementia.

As dementia has been divided into two types, mostly which occurs after cognitive impairment is Alzheimer's disease. This disease has become very common with elderly people(which are above 65) which is called later onset of Alzheimer's disease(Load).In the recent years this disease is growing like leaps and bounds in the whole world[25].

REALATED WORK

\begin{tabular}{|c|c|c|}
\hline Year & Proposed Solution & Reference \\
\hline 2016 & $\begin{array}{l}\text { gave a clear picture of contribution or current trends in usage of machine } \\
\text { learning algorithms in predicting the transformation of mild impairment to } \\
\text { Alzheimer is depicted. In it various databases like Pubmed ,Scopus and Web } \\
\text { of Science were explored and then relevant data was extracted to reach the } \\
\text { Final goal for presenting the inclination of machine learning algorithms. }\end{array}$ & [19] \\
\hline 2016 & $\begin{array}{l}\text { proposed a system which deals with diagnosis of Alzheimer's disease. To } \\
\text { diagnose the disease three parts of brain is considered frontal, Sagittal and } \\
\text { Axial. Dataset of } 19-20 \text { taken in this paper was from OASIS (Open Access } \\
\text { Series of Imaging Studies).It reached higher accuracy rate in diagnosing the } \\
\text { disease and for future prospects longitudinal monitoring application needs to } \\
\text { be added to it. }\end{array}$ & [9] \\
\hline 2017 & $\begin{array}{l}\text { proposed the concept of abnormal behaviour factors of a human like forgetting } \\
\text { and repeating activities, Dehydration and disruption in sleep etc. Methods like } \\
\text { RNN was used for activity detection in comparison to other models which } \\
\text { showed that RNN out powered all other models in detection of abnormal } \\
\text { behaviour. }\end{array}$ & [9] \\
\hline 2017 & $\begin{array}{l}\text { proposed a method in which various filters were applied on data using } \\
\text { Convolutional network and then reached at an accuracy level of } 73.75 \% \text { in } \\
\text { detection of Alzheimer's disease.In this paper Brain MRI were used for } \\
\text { detection and for diagnosing and evaluating of data less than an hour was } \\
\text { taken by the proposed system }\end{array}$ & [4] \\
\hline 2018 & $\begin{array}{l}\text { proposed a technique which analysed MRI dataset that reduced grey matter } \\
\text { volume shrinks the brain and ventricle size discriminates between person with } \\
\text { Alzheimer's disease and Normal person. }\end{array}$ & [5] \\
\hline 2018 & $\begin{array}{l}\text { nalysed the paper and has divided the paper into three areas i)diagnosis-Using } \\
\text { statistical techniques biomarkers of the disease was taken out ii)Survival } \\
\text { analysis- Using analytical techniques survival rate after onset of disease is } \\
\text { analysed. Iii)Disease Progression- using estimation techniques transition state } \\
\text { of patient is estimated. }\end{array}$ & [7] \\
\hline 2018 & $\begin{array}{l}\text { has shown analysis by using three machine learning algorithm and their } \\
\text { accuracy in detection of dementia. Brain MRI data set was collected from } \\
\text { OASIS on which classifier techniques using WEKA tool was applied and then }\end{array}$ & [3] \\
\hline
\end{tabular}




\begin{tabular}{|l|l|l|}
2 & finally classification accuracy was calculated to detect dementia. & \\
\hline 2018 & $\begin{array}{l}\text { proposed a method called ensemble support vector machine(SVM) which is a } \\
\text { combination of different SVM altogether. On the basis of MRI and mini } \\
\text { mental state examination various machine learning methods were } \\
\text { compared.SVM is experimented with linear kernel and radial function to } \\
\text { classify into normal, Mild cognitive impairment, conversion to Mild cognitive } \\
\text { impairment and to Alzheimer's disease }\end{array}$ & {$[3]$} \\
\hline 2019 & $\begin{array}{l}\text { proposed a system in which Speech recording is done of the patient. Acoustic } \\
\text { features are extracted to detect whether a person fall under the category of } \\
\text { neurodegenerative dementia or functional memory disorder. }\end{array}$ & {$[2]$} \\
\hline 2019 & $\begin{array}{l}\text { deals in extraction of feature using Segmentation based fractional texture } \\
\text { analysis(SFTA).In SFTA data was extracted from 50 demented and 50 non- } \\
\text { demented subject MRI by breaking of image into binary image. On the basis } \\
\text { of feature extraction classification for diagnosis is done using feed forward } \\
\text { neural network. Neural network gave accuracy of 97.5\%. }\end{array}$ & {$[4]$} \\
\hline
\end{tabular}

\section{PROPOSED WORK}

\section{TRANSFER LEARNING MODULE}

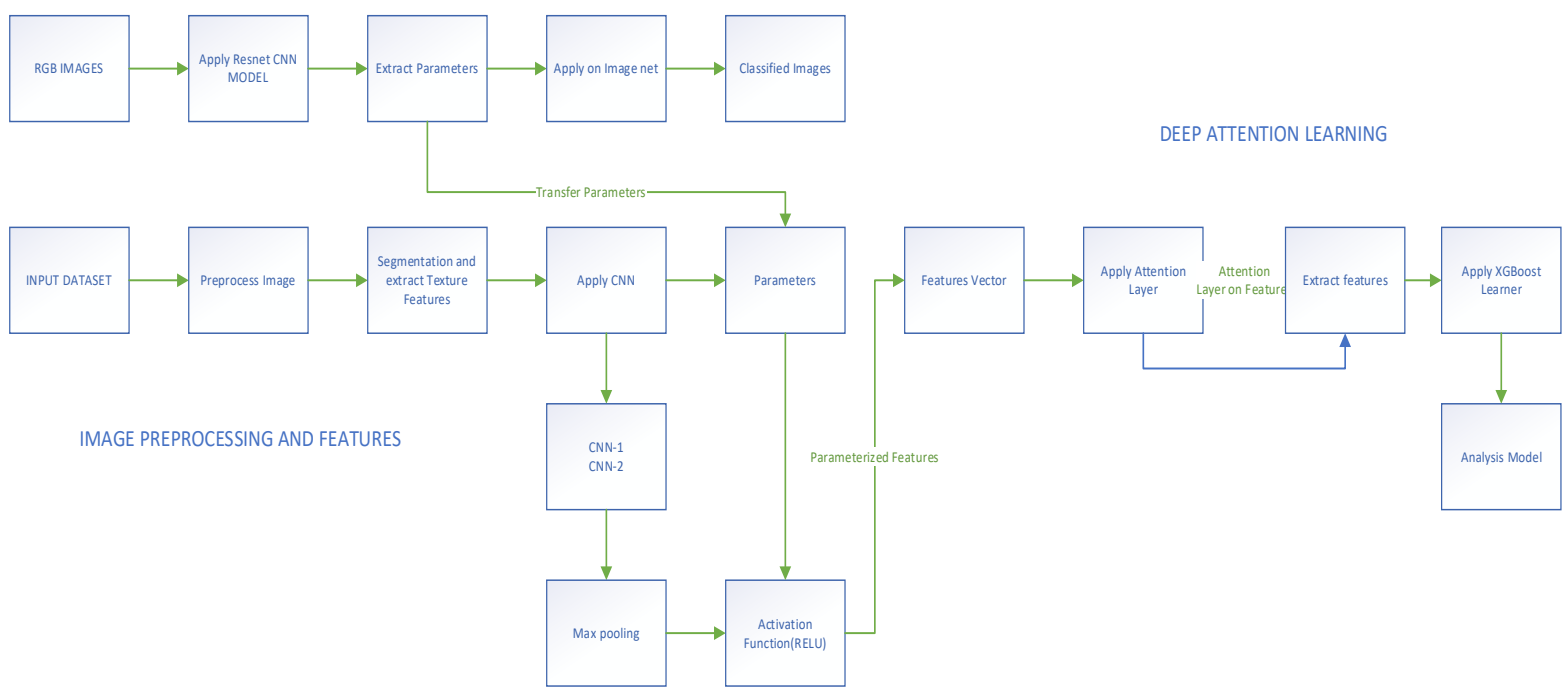

DEEP CONVLUTION MODULE

Fig1: Proposed Workflow chart

\section{TRANSFER LEARNING}

In this phase, CNN model-based feature extractor is designed as shown in Figure 2 using RESnet architecture and transfer learning method [4,5]. The parameters of the CNN model (RESnet50) pertain to a natural image set known as ImageNet. It is utilized to extract the low-level features from the generated ROI of mammogram images. The structure of the CNN model in transfer learning consisted of four convolution layers (conv) of RESnet architecture with a ReLU mapping activation function and a simple fully connected layer (FC) as shown in Table 1. The transfer learning method fine-tuned the pre-trained $\mathrm{CNN}$ model and generated the feature vectors for mass classification. The output of the pre-trained CNN model is low-level features. 
Table 1. CNN Model for Transfer Learning

\begin{tabular}{|c|c|c|}
\hline \multirow{16}{*}{ 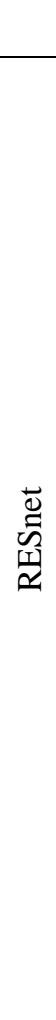 } & \multicolumn{2}{|c|}{ Input : Image } \\
\hline & \multirow{3}{*}{$\begin{array}{c}\text { Conv Block } 1 \\
(4 * 48 * 64) \\
\text { Pooling } \\
(2 * 24 * 64)\end{array}$} & Conv + ReLU \\
\hline & & Patch $7 * 7$ \\
\hline & & Max Pool $3 * 3$ \\
\hline & \multirow{3}{*}{$\begin{array}{c}\text { Conv Block } 2 \\
(2 * 24 * 64)\end{array}$} & Conv + ReLU 1*1(64) \\
\hline & & Conv $+\operatorname{ReLU} 3 * 3(256)$ \\
\hline & & Conv $+\operatorname{ReLU} 1 * 1$ (512) \\
\hline & \multirow{3}{*}{$\begin{array}{c}\text { Conv Block } 3 \\
(2 * 24 * 64)\end{array}$} & Conv $+\operatorname{ReLU} 1 * 1$ (128) \\
\hline & & Conv +ReLU 3*3 (128) \\
\hline & & Conv +ReLU 1*1 (512) \\
\hline & \multirow{3}{*}{$\begin{array}{c}\text { Conv Block } 4 \\
(2 * 24 * 64)\end{array}$} & Conv + ReLU 1*1 (256) \\
\hline & & Conv +ReLU 3*3 (256) \\
\hline & & Conv +ReLU1*1 (1024) \\
\hline & \multirow{3}{*}{$\begin{array}{l}\text { Conv Block } 5 \\
(2 * 24 * 64)\end{array}$} & Conv $+\operatorname{ReLU} 1 * 1$ (512) \\
\hline & & Conv $+\operatorname{ReLU} 3 * 3$ (512) \\
\hline & & Conv +ReLU1*1 (2048) \\
\hline \multicolumn{3}{|c|}{ FC + ReLU (Dropout= 0.5) } \\
\hline \multicolumn{3}{|c|}{ Output (Sigmoid):[0,1] } \\
\hline
\end{tabular}

\section{CONVOLUTION WITH ATTENTION LAYER}

In this phase, the features of $\mathrm{CNN}$ and attention models are combined. Both models are worked parallel for extracting the features from the input mammogram image. CNN integrated CNN Extracted Features which were fitted together with a thick sheet. attention learning for better function collection. In order to conform to various document sizes, the mechanism automatically selects features of different scales at each location in the text. As the focus function tests weight distribution, it estimates an array. $X_{i}, f\left(X_{i}, X_{i+1}\right)$ features from the second layer and $f\left(X_{i}, X_{i+1}, \cdots, X_{i}+L-1\right)$ from the L-th layer. These feature values indicate the responses of multi-scale ngrams, i.e., unigram $X_{i}$, bigram $X_{i} X_{i+1}$. and L-gram $X_{i} X_{i+1} \cdots X_{i}+L-1$. In the focus mechanism, the filter ensemble and reweight scale are used in unison. a scale-descriptor filter is generated to feature features at each scale $X_{i} 1$ at position $i$. Scale reweight computes SoftMax distribution of attention weights using the descriptors as data, and outputs weighted attribute weights for reweighing.

$$
\begin{gathered}
S_{l}^{i}=F L_{\text {ensm }}\left(X_{l}^{i}\right) \ldots \\
X_{\text {atten }}^{i}=\sum_{j=i}^{L} \alpha_{L}^{i} X_{L}^{i}
\end{gathered}
$$

Classifier Model

$$
\partial_{i}^{L}=\operatorname{Softmax}\left(M L P\left(X_{a t t e n}^{i}\right)\right)
$$

\section{CNN MODEL}

In this phase, the CNN model is designed to extract the relevant features from the M feature matrix. The detail about the CNN model is described in Fig.4. It has four convolution layer along with two max-pooling layers and one fully connected layer. The convolution layer contains many neurons. These neurons are connected with each other's and share biases and weights. This layer converts the matrix features into the feature map by utilizing convolution operations[26]. Although, the input feature matrix is mapped with kernel sets. These kernels produce the new feature map $F_{u}$ and this procedure is known as convolution. The value of feature at location (s, d) in the uth feature map of kth layer, the value of $C_{u}^{k}$ is computed by using equation 15 . 


$$
C_{u}^{k}(s, d)=w_{u}^{k} I^{k}(s, d)+b_{u}^{k}
$$

The max pooling is used to reduce the computed feature maps dimension. ReLU activation function is used in each layer expect the last output layer, for that sigmoid function is used. After the 4-convolution layer and 2 max pooling operation, the output of final convolution layer is propagated into the fully connected layer. Let the output of final convolution layer is $E^{g}$ and it is calculated by using equation 16 .

$$
E^{g}=f\left(I^{g}\right)=f_{\text {sigmoid }}\left(\sum_{u} w_{u} * f\left(I^{g-1}\right)\right)
$$

Where, $\mathrm{u}$ is the kernel numbers that are utilized in final convolution layer, $w_{u}$ represents the kernel weight, $f\left(I^{g-1}\right)$ is the value of activation function of $(g-1)$ convolution layer. Finally, the fully connected layer contains the feature vectors with labels. These feature vectors are fed into ensemble learning model for breast mass classification.

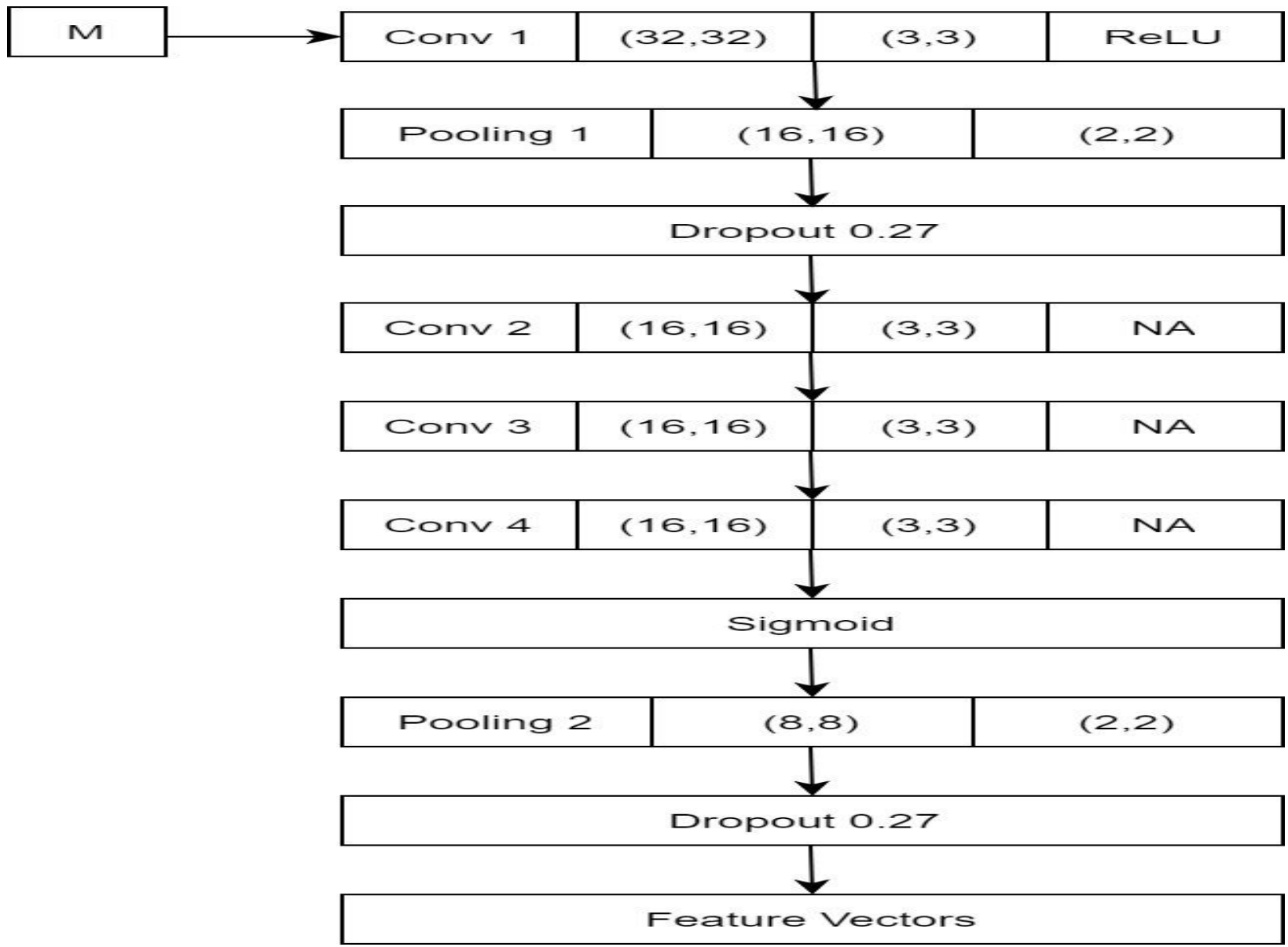

Fig2 . Proposed CNN Architecture

\section{ENSEMBLE LEARNING MODEL FOR CLASSIFICATION}

In this phase, the ensemble learning-based classification model is proposed by combining extreme gradient boosting with Xg-boost technique. In the proposed system, the Xg-boost is used to generate the different regression trees from the feature vectors with labels along with different threshold values. It is not capable of taking the final prediction decision regarding the different tree combinations. Hence, Whereas, the $\mathrm{C}$ technique is used to calculate the threshold values for model selection in the testing phase. It has an effective regularization function, in turn, less value of loss function is generated by using the following objective functions $\mathrm{F}$ :

$$
F=\sum_{x=1}^{n} l\left(A_{x}, \hat{A}_{x}\right)+(\gamma T-(1-\gamma) T)+\frac{1}{2} \lambda \sum_{y=1}^{T} w_{y}^{2}
$$

\section{RESULT AND ANALYSIS \\ PERFORMANCE METRICS}

Accuracy: The appropriately categorized are listed. The accuracy of the method proposed is determined by equation 7 .

$$
\text { Accuracy }=\frac{\mathrm{P}+\mathrm{N}}{\mathrm{P}+\mathrm{N}+\mathrm{F}+\mathrm{G}}
$$


In equation $7, \mathrm{P}$ marks the samples that were accurately categorized, $\mathrm{N}$ represents the negative samples properly classified, F denotes the samples classified wrongly, and $\mathrm{G}$ denotes samples that have been properly classified as samples.

Specificity: It defines the true negatives that can be identified accurately by the diagnostic examination. The specificity is determined by equation 8 .

$$
\text { Specificity }=\frac{P}{P+F}
$$

Sensitivity: This shows real patients of both patients with illness. Calculation of sensitivity metric equation 8 .

$$
\text { Sensitivity }=\frac{P}{P+G}
$$

F-measure: It is demonstrated as expression of specificity and sensitivity. It only takes the positive samples either classified. Equation 10 is used to compute the F-measure.

$$
\mathrm{F}-\text { measure }=\frac{2 \mathrm{P}}{2 \mathrm{P}+\mathrm{P}+\mathrm{G}}
$$
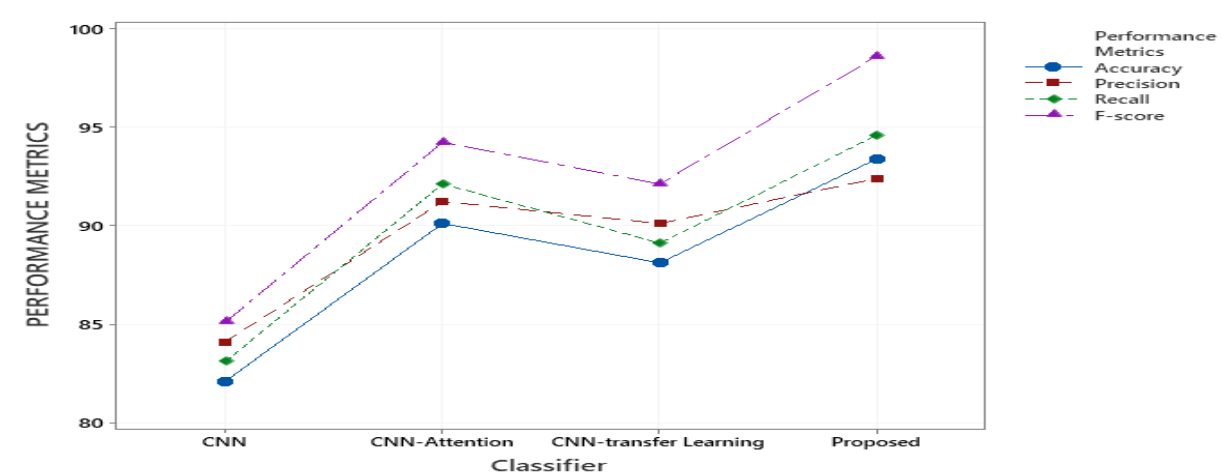

Fig3: Comparison of Performance metrics On Proposed approach and Existing Deep learning Approaches

In above fig3 comparison of proposed and other deep learning models like CNN, CNN-attention, CNN-transfer Learning by different parameters like Accuracy ,Precision ,recall and f-score. By this experiment following observation find

- In fig3 proposed approach show significantly improve all parameters like accuracy, precision and recall.

- Accuracy improve 5-6\%, Precision improve 2-3\% and recall improve 6-7\% in proposed approach.

- In proposed approach improved parameters come because of optimize tune parameters by transfer learning and attention of efficient features using attention layers.

- In proposed approach improve feature mapping learn by gradient base xg boost classifier which improve the results by ensemble and normalization of error.
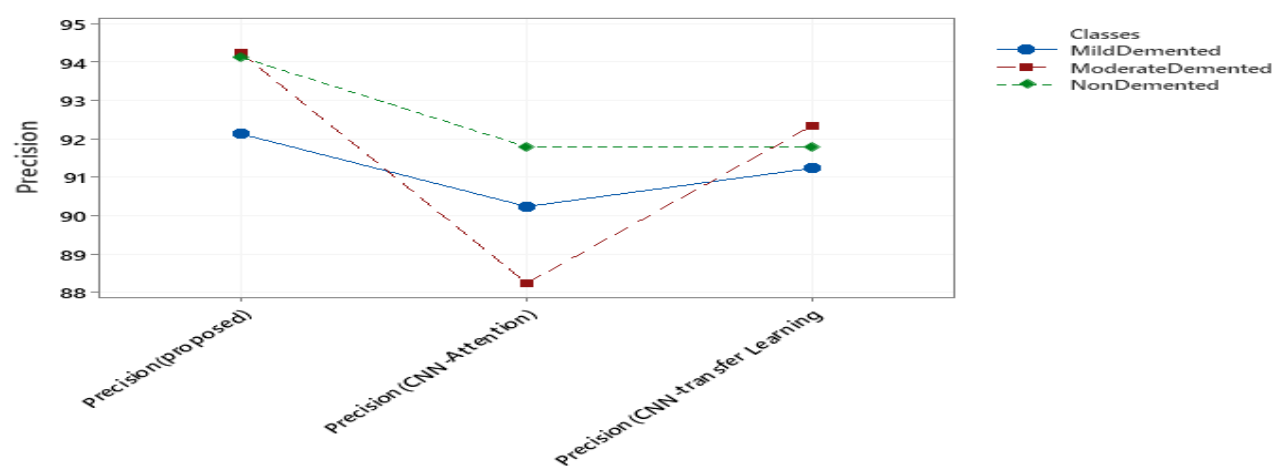

Fig4: Comparison of Precision On Proposed approach and Existing Deep learning Approaches 
In fig4 and fig5 comparison of Precision and Recall performance class wise performance. In this comparison show that proposed approach detection efficiently and reduce type 2 error by its precision and recall

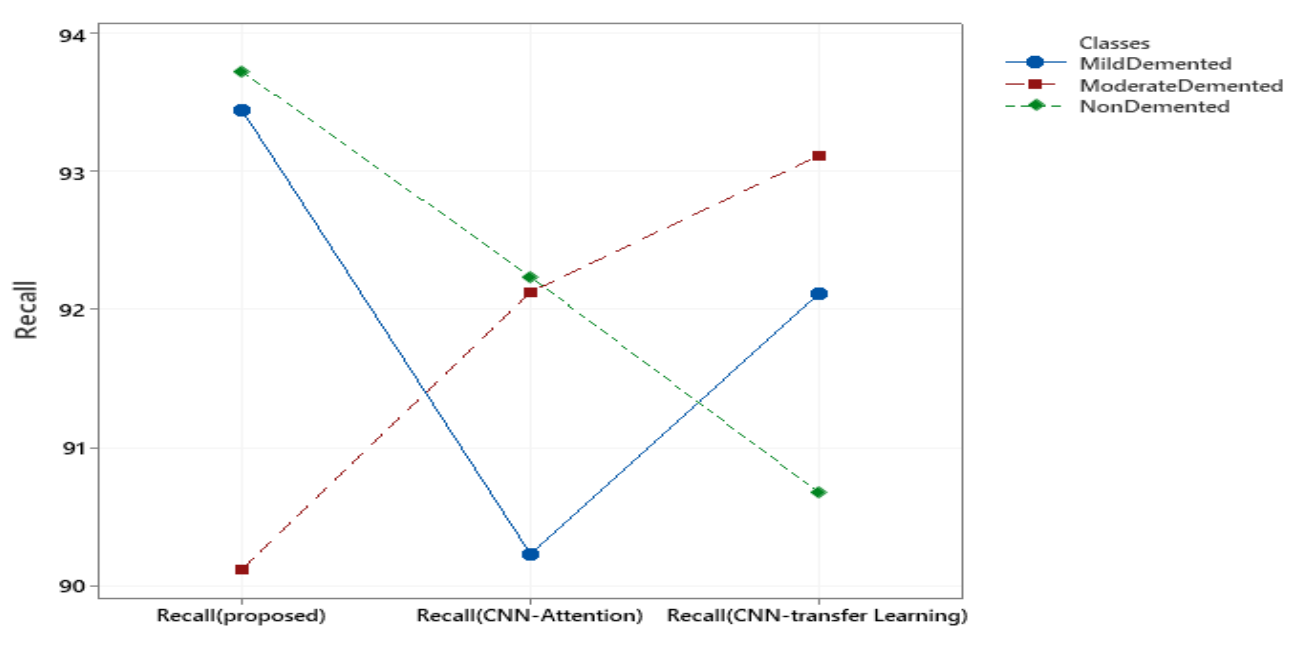

Fig5: Comparison Of Recall On Proposed approach and Existing Deep learning Approaches

\section{CONCLUSION}

The article uses a deep learning model to investigate stages of Alzheimer's disease using multi-class classification. We suggested a CNN-to-transfer mapping of attention. We describe our proposed approach with ADNI detail studies, where the MRI has four distinct categories: No dementia (ND), mild dementia (MD), moderate dementia (MMD), and very severe dementia (MSD). We conduct experiments to assess model regularization and overfitting in different classes to get a better idea of how they affect the overall performance of our application. is the approach proposed in the gradient Boost method that leverages hyperparameter variation and parameter optimization for pre-processing the Boosting framework allows poor classifier models to learn sequentially. classifier is created in the manner of performing residual classification using gradient descent. Dementia The mortality rate is significantly lower for elderly people than it is for other age groups in particular, especially those over the age of seventy. Precision increase 2-3\% and recall improving recall and recall efficiency will result in a 5-6\% improvement.

\section{REFERENCES}

Ishii, H., Kimino, K., Aljehani, M., Ohe, N. and Inoue, M. (2016). An Early Detection System for Dementia using the M2M/IoT Platform. In: 20th International Conference on Knowledge Based and Intelligent Information and Engineering Systems. [online] elsevier, pp.1332-1340. Available at: http://www.sciencedirect.com

Mirheidari, B., Blackburn, D., Walker, T., Reuber, M. and Christensen, H. (2019). Dementia detection using automatic analysis of conversations. Computer Speech \& Language, 53, pp.65-79.

Bansal, D., Chhikara, R., Khanna, K. and Gupta, P. (2018). Comparative Analysis of Various Machine Learning Algorithms for Detecting Dementia. Procedia Computer Science, 132, pp.1497-1502.

Chen, j., zhang, j., harvey, a. and Shue, L. (2005). An automatic Acoustic bathroom Monitoring system. In: IEEE international symposium on circuits and systems. IEEE.

Nergui, M., Komekine, k., Nagai, H. and Otake, M. (2014). Development of laughter motion on the cognitive robot "Bono-02"Assisting group Conversation. In: 7th International Conference on Ubi-Media Computing and workshops. IEEE.

Patterson, C. (2018). Alzheimer 's disease International: World Alzheimer Report 2018. [Online] London: Alzheimer's Disease International (ADI), London., p.48. Available at: https://www.alz.co.uk/research/WorldAlzheimerReport2018.pdf

Deo, V. (2018). Prospects of Statistical and Biostatistical Techniques in the Study of Diagnosis, Survival Analysis, and Disease Progression of Alzheimer's Disease. Biostatistics and Biometrics Open Access Journal, [online] Volume 7(3 - July 2018). Available at: https://juniperpublishers.com/bboaj/pdf/BBOAJ.MS.ID.555712.pdf

Arifoglu, D. and Bouchachia, A. (2019). Detection of Abnormal Behaviour for Dementia Sufferers using Convolutional Neural Networks. artificial intelligence in medicine. doi:10.1016/j.artmed. 
Arifoglu, D. and Bouchachia, A. (2017). Activity Recognition and Abnormal Behaviour Detection with Recurrent Neural Networks. In: 14th International Conference on Mobile Systems and Pervasive Computing (MobiSPC 2017). [online] Available at: http://Procedia Computer Science

Umphred, D. and Lazaro, R. (2012). Umphred's neurological rehabilitation. 6th ed. Elsevier, p.1280.

Abe, Y., Toya, M. and Inoue, M. (2013). Early Detection System of Senile Dementia by Behaviour Sensing. In: IEEE International Symposium on Consumer Electronics (ISCE). IEEE, pp.67-68.

Tohka, J., Moradi, E. and Huttunen, H. (2016). Comparison of Feature Selection Techniques in Machine Learning for Anatomical Brain MRI in Dementia. Neuroinformatics, 14(3), pp.279-296.

Batista, E., Borras, F., Casino, F. and Solanas, A. (2015). A study on the detection of wandering patterns in human trajectories. In: 2015 6th International Conference on Information, Intelligence, Systems and Applications (IISA). Corfu, Greece: IEEE.

Pavisic, I., Firth, N., Parsons, S., Rego, D., Shakespeare, T., Yong, K., Slattery, C., Paterson, R., Foulkes, A., Macpherson, K., Carton, A., Alexander, D., Shawe-Taylor, J., Fox, N., Schott, J., Crutch, S. and Primativo, S. (2017). Eyetracking Metrics in Young Onset Alzheimer's Disease: A Window into Cognitive Visual Functions. Frontiers in Neurology, 8.

Dolatabadi, E., Zhi, Y., Flint,, A., Mansfield, A., Iaboni, A. and Taati, B. (2019). The feasibility of a visionbased sensor for longitudinal monitoring of mobility in older adults with dementia. Archives of Gerontology and Geriatrics. [online] Available at: http://www.sciencedirect.com/science.

Weakley, A., Williams, J., Edgecombe, M. and Cook, D. (2015). Neuropsychological test selection for cognitive impairment classification: A machine learning approach. Journal of Clinical and Experimental Neuropsychology, [online] 37(9), pp.899-916. https://www.tandfonline.com/loi/ncen20

Jammeh, E., Carroll, C., Pearson, S., Escudero, J., Anastasiou, A., Zhao, P., Chenore, T., Zajicek, J. and Ifeachor, E. (2018). Machine-learning based identification of undiagnosed dementia in primary care: a feasibility study. BJGP Open, pp.bjgpopen18X101589.

Rodríguez, L., Salazar, D., García, N., L. Pastor, L. and Guinea, Ó. (2018). Magnetic resonance imaging in dementia. Radiología. 2018, [online] 60(6), pp.476-484. Available at: http://www.sciencedirect.com/science/article/pii/S2173510718300533?via\%3Dihub

Dallora, A., Eivazzadeh, S., Mendes, E., Berglund, J. and Anderberg, P. (2016). Prognosis of dementia employing machine learning and microsimulation techniques: a systematic literature review. In: Conference on Enterprise Information Systems/International Conference on Project management / Conference on Health and Social Care Information Systems and Technologies, CENTERIS. [online] Elsevier, pp.480 - 488. Available at: http://www.sciencedirect.com

Pellegrini, E., Ballerini, L., Hernandez, M. del C. V., Chappell, F. M., González-Castro, V., Anblagan, D.et.al (2018). Machine learning of neuroimaging for assisted diagnosis of cognitive impairment and dementia: A systematic review. Alzheimer's \& Dementia: Diagnosis, Assessment \& Disease Monitoring.doi:10.1016/j.dadm.2018.07.004

Ahmed, M. R., Zhang, Y., Feng, Z., Lo, B., Inan, O., \& Liao, H. (2018). Neuroimaging and Machine Learning for Dementia Diagnosis: Recent Advancements and Future Prospects. IEEE Reviews in Biomedical Engineering, 1-1.doi:10.1109/rbme.2018.2886237

Dyrba, M.et al. (2013). Robust Automated Detection of Microstructural White Matter Degeneration in Alzheimer's Disease Using Machine Learning Classification of Multicenter DTI Data. PLOS ONE, 8(5).

Bhagyashree, S., Nagaraj, K., Prince, M., Fall, C. and Krishna, M. (2017). Diagnosis of Dementia by Machine learning methods in Epidemiological studies: a pilot exploratory study from south India. Social Psychiatry and Psychiatric Epidemiology, 53(1), pp.77-86.

Guram, H. and Sharma, A. "Identifying the Rules of Transition from AD to Dementia and MCI Using Machine Learning", International Journal of Control and Automation, vol. 13, no. 4, pp. 718-729, 2020.

Guram, H. and Sharma, A. "Patch base Segmentation for Classification of Dementia disorder with optimize feature weight and Random forest based approach", Materials Today: Proceedings, 2020.

Guram, H. and Sharma, A."Review Paper on Detection of Dementia related disorders Among Aged People", International Journal Of Research In Electronics And Computer Engineering (Ijrece), vol. 7, no. 3, pp. 478-482, 2019. [Accessed 15 December 2020]. 